\title{
GIS-based analysis of urban traffic model: Case study in Beijing
}

\author{
Yongkai Liang ${ }^{1, *}$, Jingyuan $\mathrm{Li}^{2}$, and Hai Liu ${ }^{1}$ \\ ${ }^{1}$ School of Mechanical Engineering, Hebei University of Technology, Tianjin \& 300132, China \\ ${ }^{2}$ China Automotive Technology and Research Center Co., Ltd., Tianjin \& 300300, China
}

Keywords: GIS, traffic flow model, traffic flow, Vehicle travel time, roads of all levels.

\begin{abstract}
Taking the traffic flow characteristics of Beijing's entire road network as the object, and using the low-frequency traffic big data of GIS (Geographic Information System), the roads of the whole road network are divided into four road grades, and the traffic flow-speed models are constructed respectively. In view of the deviation of the model calculation caused by the sudden rise and fall of the traveling vehicle at night, the flow of the traffic flow model is corrected by cubic polynomial fitting, and the mathematical model is compared, calibrated and verified. Focus on analyzing the influence of roads of different grades and seasons on the characteristics of road traffic flow, and provide data support for further research on intelligent transportation.
\end{abstract}

\section{Introduction}

With the increasing complexity of urban transportation, a single indicator can no longer meet the evaluation of my country's transportation diversification. The mining of transportation big data came into being, which helps car users to understand the driving situation of urban roads, and also provides data support for improving the service level of urban roads and the distribution of transportation modes of transportation networks.

Due to the difficulty of conducting a comprehensive traffic flow survey in a large area, the workload is large, and it is difficult to promote, so the traffic flow model is usually used to estimate the traffic flow of the actual road, so as to analyze the traffic flow characteristics of the roads of all levels. Big data, as an emerging technical means, can make up for the shortcomings of the traditional methods of sampling data subjectively influenced by more subjective, more comprehensive analysis of road traffic flow characteristics ${ }^{[1]}$. Since the collected big data information only contains the vehicle speed and time, it is impossible to directly obtain the traffic information of the entire road network. Therefore, it is necessary to construct a traffic flow-speed model to indirectly obtain the traffic data of the entire road network.

In the past few decades, many researchers have proposed various traffic flow theories and models to calculate traffic flow and speed distribution ${ }^{[2-4]}$. In 1935, the sample size of the

\footnotetext{
*Corresponding author: liangyongkai1113@163.com
} 
traffic flow model proposed by Greenshields was insufficient, and it was not suitable for studying highway traffic flow and high density traffic flow ${ }^{[5]}$. The logarithmic speed-density relationship model proposed by Greenberg in 1959 is suitable for traffic congestion, but it is not suitable for free flow traffic ${ }^{[6]}$. In 1961, Underwood proposed a speed-density index model for low-density traffic flow. So far, the research on the characteristics of traffic flow conducted by scholars at home and abroad has mainly focused on expressways and expressways ${ }^{[7]}$. Due to the influence of traffic lights, pedestrians and other complex factors, there are few studies on the traffic flow model of the whole road network at home and abroad. This paper builds a flow-speed model based on GIS traffic flow big data, graded roads, and corrects the flow by performing multiple term fittings for the sudden rise and fall of speed at night. And on this basis, the analysis of road grades, seasonal changes on traffic flow characteristics.

\section{Construction of traffic flow model}

\subsection{Selection of traffic flow model}

The traffic flow model estimates traffic flow based on driving speed and is a function of driving speed and traffic density, so it is also called a traffic flow-speed-density model, as shown in Equation 1.

$$
q=u \cdot k
$$

In the formula, $q$ represents the flow rate, which refers to the number of vehicles passing a certain point, a certain section or a certain lane of the road in the selected time period, the unit is $\mathrm{veh} / \mathrm{h}^{[8]}$.

$u$ represents vehicle speed, which is an important parameter among the three parameters of traffic flow, that is, the distance traveled by the vehicle per unit time, in $\mathrm{km} / \mathrm{h}$.

$k$ represents the traffic density, the number of vehicles existing per unit of road length at a time, the unit is veh/ $\mathrm{km} / \mathrm{lane}$.

The traffic situation of each city has its own characteristics. When constructing a traffic flow model, the basic traffic flow mechanism should be followed, and the model should be established according to the distribution of measured data. The model used in this paper is the Underwood velocity-flow model, which is characterized by a simple structure, easy calibration, and flexibility. Underwood's exponential model expression 2 proposed for lowdensity traffic flows is shown as follows:

$$
v=v_{f} e^{-\frac{\rho}{\rho_{m}}}
$$

Combined with formula 1 , the flow-speed model is obtained after pushing to finishing:

$$
q=\rho_{m} v \ln \frac{v}{v_{f}}
$$

where $v_{f}$ is the free flow speed, that is, when the density is 0 , the vehicle speed can reach the theoretical maximum speed.

$\rho_{m}$ is the density corresponding to the maximum flow rate, which is called the optimal density.

The parameters to be calibrated for the traffic flow model are the optimal density and the free flow speed. The free flow speed is calibrated using the percentile of the speed data, and the speed quantiles of different grades of roads are different. The expressway is calibrated according to the speed limit classification. The determination of the optimal density is related 
to the size of the city and the commuting ability of roads at various levels, and the calibration of the optimal density can be completed by a heuristic algorithm.

\subsection{Modification of traffic flow model}

\subsubsection{Minimum flow correction}

Calculate the flow of single lanes of roads of various grades, according to the rules of traffic flow, specify the minimum flow of single lanes of roads of various grades, and correct the minimum flow of the calculation results.

Table 1. Minimum flow of roads of various grades.

\begin{tabular}{ll}
\hline Road type & Minimum flow(veh/h) \\
\hline highway & 190 \\
Freeway & 43 \\
Main road & 23 \\
Secondary branch & 20 \\
\hline
\end{tabular}

\subsubsection{Night and morning traffic flow correction}

There is a sudden drop in the speed at 6:00 and 22:00. The speed change is shown in Fig. 1.

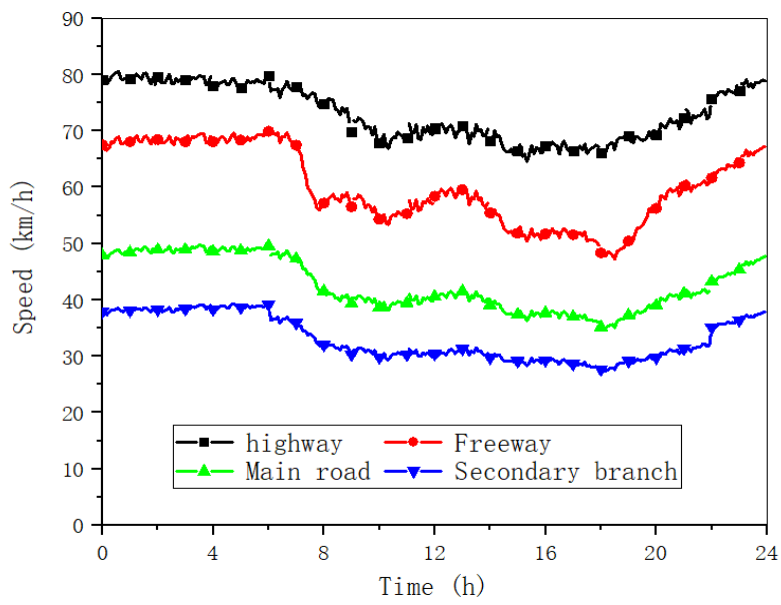

Fig. 1. Time series of road speed.

Due to the instability of the speed data, the calculated flow of the expressway and expressway in the early morning and night models does not match the actual situation, and the flow needs to be corrected according to the actual situation. This article uses cubic polynomials to adjust the freeway and expressway traffic at night and morning based on actual traffic trends. The revised formulas are shown in Equations 4-7 in order:

$$
\begin{aligned}
& S Q=3.8139 t^{3}-37.697 t^{2}+101.27 t+156.92 \\
& S Q=-7.7723 t^{3}+548.9 t^{2}-12895 t+101003 \\
& S Q=5.694 t^{3}-34.667 t^{2}+54.276 t+188.39 \\
& S Q=-0.313 t^{3}+61.512 t^{2}-2362.1 t+25756
\end{aligned}
$$


In the formula, $\mathrm{SQ}$ is the single-lane flow, and $\mathrm{t}$ is the flow correction time.

\subsection{Validation of traffic flow model}

In order to verify the accuracy of the model, it is necessary to compare the calculation results of the model with the actual results. The actual data used for verification is the actual survey data in 2018, and the survey methods are all manual counting methods. Due to the high speed of the highway, it is difficult to obtain traffic flow data, so only the freeway (North Fourth Ring Road and East Second Ring Road), main road (Chaoyang North Road and Fangzhuang Road), secondary branch road were selected as typical roads (Baiziwan Road and Guang'an Road), the relative error and absolute error of the model flow and the field survey flow were calculated. The results are shown in Tab.2.

Table 2. Comparison of model traffic and field survey traffic.

\begin{tabular}{lll}
\hline Research section & Absolute error & relative error \\
\hline Expressway (North Fourth Ring Road) & 157.59 & 0.1217 \\
Expressway (East Second Ring Road Main Road) & 130.91 & 0.1065 \\
Main Road (Fangzhuang Road) & 90.31 & 0.1393 \\
Main Road (Chaoyang North Road) & 86.24 & 0.1469 \\
Secondary Road (Baiziwan Road) & 113.18 & 0.1435 \\
Secondary Branch Road (Ganging Road) & 102.04 & 0.1167 \\
\hline A
\end{tabular}

According to Fig. 2, the traffic flow calculated by the expressway, expressway, and fastroute traffic flow models is consistent with the survey flow trend, with a small difference, and the model is more accurate. 

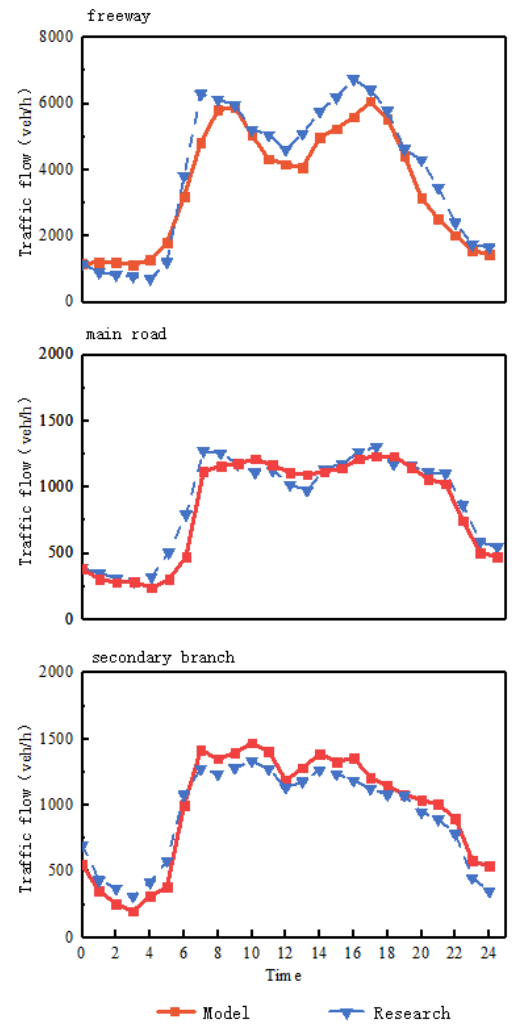

Fig. 2. Comparison of model traffic and research traffic.

\section{Analysis of traffic flow characteristics based on flow}

\subsection{Analysis of traffic flow characteristics of Beijing's entire road network}

\subsubsection{Analysis of various levels of roads in Beijing}

The roads of all levels undertake different carrying tasks in urban transportation. The expressway is responsible for communication between cities; expressways are mainly used for commuting transportation in the city in the morning and evening, and are less set up in the urban road network; main roads are used to connect the main divisions and are the aorta of the city; The travel is closely related, is the connecting line between the neighborhoods. The length of roads at various levels is shown in Fig. 3. The total length of main roads and sub-branch roads is relatively high, and assumes the main transportation tasks of the city. Different grades of roads carry different road network transportation tasks, and the proportion of length is obviously different. Therefore, it is necessary to analyze the influence of each grade of roads on the characteristics of Beijing's traffic flow. 


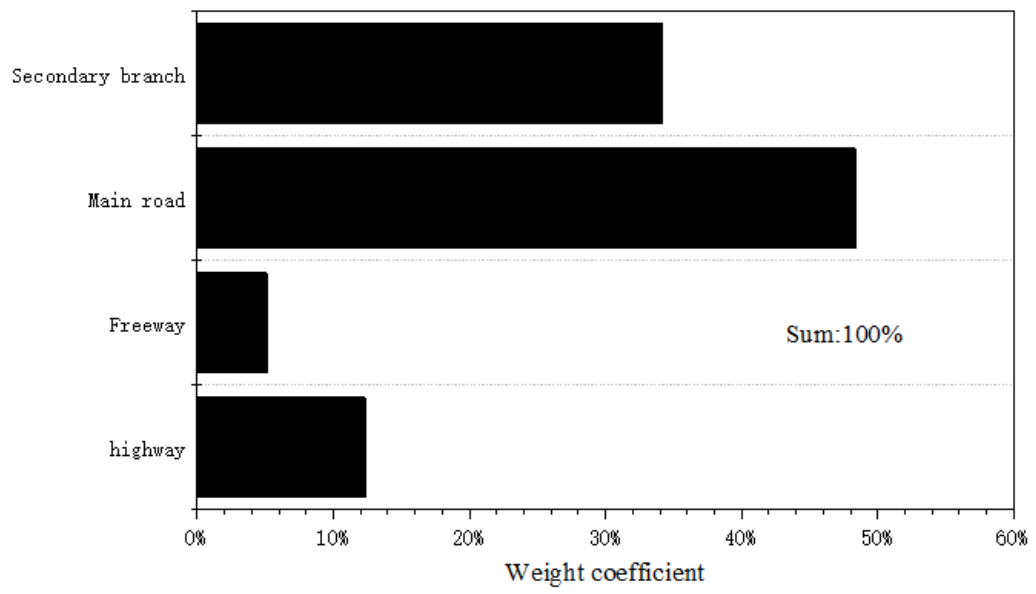

Fig. 3. Proportion of road lengths of various grades in Beijing.

\subsubsection{Analysis of traffic flow of various grades in Beijing}

The time-varying trend of road traffic flow at various levels is shown in Fig. 4, showing a distinct "saddle" type, which can also be referred to as an "M" type. There are morning and evening peaks and noon peaks. The duration of the morning rush hour is about 7:00-9:00; the duration of the evening rush hour is about 17:00-19:00; the traffic flow at noon (11:00-13:00) will slightly decrease, People generally choose to eat and rest, so road traffic will show a downward trend. The traffic flow is stable from 22:00 to 6:00 the next day, the road operation is in a free flow state, and the road operation state is the best.

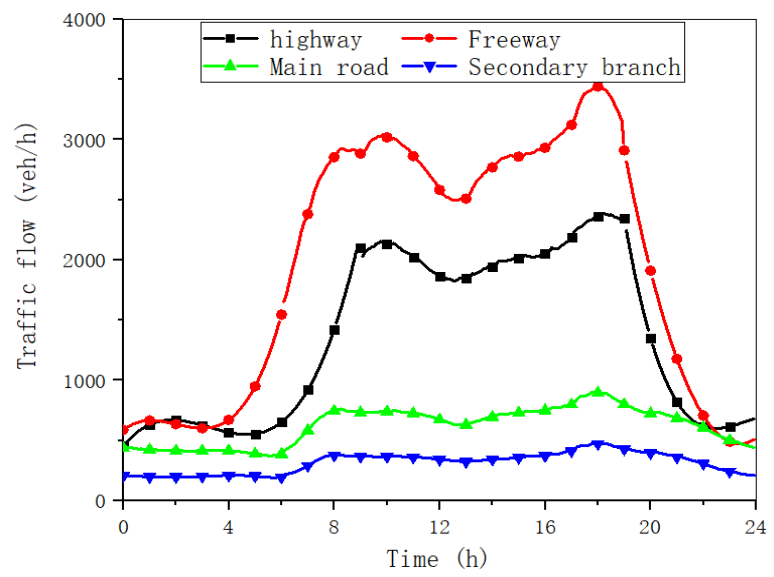

Fig. 4. Time series of road traffic in Beijing.

The time-varying curve of the average traffic flow of the expressway shows obvious double peaks, and the formation time of the night peaks is long and the speed of retreat is slow. According to the average flow chart of roads, it is found that the speed fluctuation is small, the road operation is relatively stable, and there is a "lag" phenomenon. Expressway and main roads have obvious road fluctuations during the day, and there will be a significant drop in traffic at noon. The expressways and main roads are mainly responsible for carrying 
traffic to and from roads in the city. There will be a decrease in traffic flow during noon breaks, and road operating conditions will improve. It has obvious characteristics of tidal commuting.

\subsubsection{Analysis of speed distribution in Beijing}

Vehicle Hours Traveled (VHT) is the product of the average traffic flow on the road segment and the average travel time of the vehicle. It includes the dual effects of road length and road congestion, which is closer to the actual traffic demand. It can not only reflect the traffic congestion, but also reflect the traffic demand of traffic travelers. In a certain period of time, the calculation method of VHT for a road section is:

$$
\operatorname{VHT}_{i}=q_{i} \times T_{i}
$$

$V H T_{i}$ represents the travel time of the vehicle on the road segment $i$ at a certain time:

$q_{i}$ means the average traffic flow of road segment i at a certain time;

$T_{i} \quad$ means the average travel time of the vehicle on a certain road section $\mathrm{i}$;

Calculate the VHT of different grades of roads at different times throughout the day, cluster analysis of VHT according to the corresponding speed value, and obtain the speed distribution curve of Beijing's entire road network as shown in Fig. 5, with $2 \mathrm{~km} / \mathrm{h}$ as a speed interval, a total of 65 Speed interval. Judging from the distribution range (accumulated distribution of $10 \%$ and $90 \%$ corresponding speed range), the speed is mainly distributed in $22-52 \mathrm{~km} / \mathrm{h}$. Taking the middle value of the vehicle speed section as the average vehicle speed based on the calculation probability, the average speed of the sample is calculated to be $34.46 \mathrm{~km} / \mathrm{h}$ through the speed distribution statistics of Beijing's entire road network.

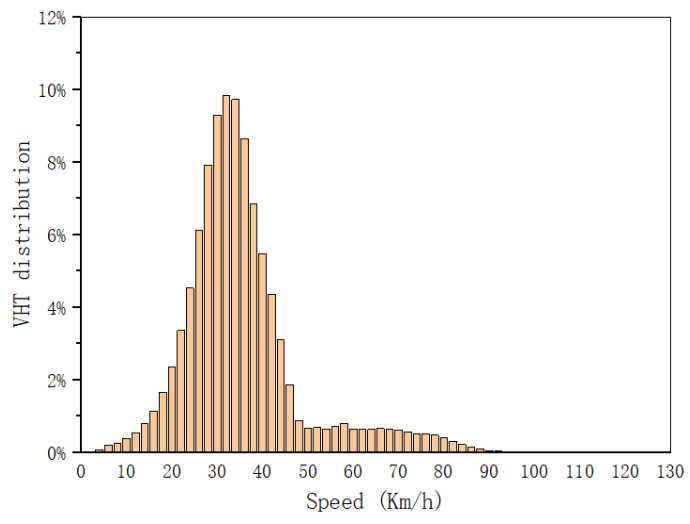

Fig. 5. Distribution of VHT in Beijing.

\subsection{Research on the influence of roads of different grades on the characteristics of traffic flow}

Refine the grade of the road network and focus on the time-varying characteristics of road traffic of various grades. It can be found that the average flow of various grades of roads has obvious differences. The expressway has a single grade, which is significantly higher than other grades. Road traffic is approaching.

The time-varying curve of the average traffic flow of the expressway shows obvious double peaks, and the formation time of the night peaks is long and the speed of retreat is slow. The expressway and the main road are mainly responsible for carrying tasks to and 
from the road in the city. During the noon rest period, there will be a decrease in traffic flow, an increase in the road operating conditions, and obvious characteristics of tidal commuting.

The VHT data of each grade of road subdivision speed interval calculated by the traffic flow model is summarized to obtain the speed distribution of each grade of road, and the speed distribution characteristics based on different road types are further analyzed, as shown in FIG. 6. There are obvious differences in the distribution of road speeds of various grades. The speed distribution of expressways is more scattered and the curve is flatter; the other road types are all positively skewed.

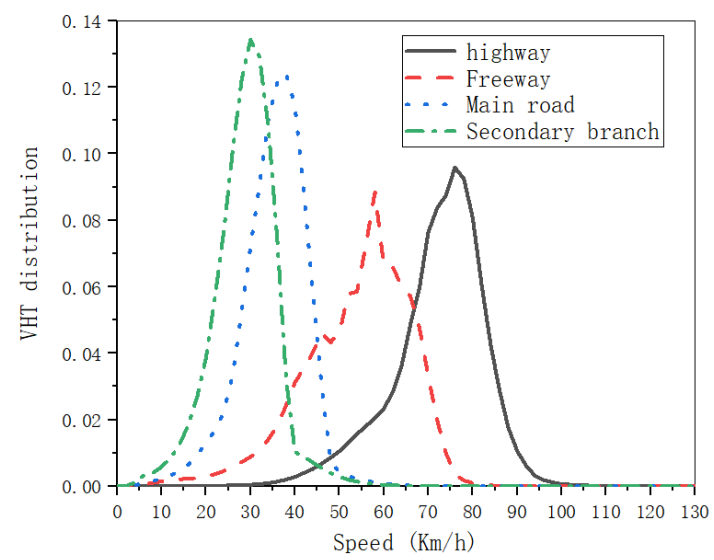

Fig. 6. Distribution of VHT of various grades of roads in Beijing.

The distribution of VHT on expressways and expressways tends to be in the high-speed section, with sample averages of $71.21 \mathrm{~km} / \mathrm{h}$ and $52.82 \mathrm{~km} / \mathrm{h}$ respectively, and the distribution of vehicle speeds on expressways is more dispersed. Among them, the main distribution range of expressways (the cumulative distribution of $10 \%$ and $90 \%$ corresponding speed ranges) is in the range of $58-84 \mathrm{~km} / \mathrm{h}$, and the main distribution range of expressways is in the range of $38-68 \mathrm{~km} / \mathrm{h}$; the distribution of VHT on main roads and secondary branches is biased In the middle and low speed range, the main speed of the main road is distributed in the range of $26-44 \mathrm{~km} / \mathrm{h}$, and the main speed of the secondary branch is distributed in the speed range of $20-36 \mathrm{~km} / \mathrm{h}$. The distribution from narrow to wide is as follows: secondary branch road $<$ main road $<$ highway road $<$ freeway road.

In summary, the road network of the whole road network is subdivided into road grades, and the traffic flow characteristics such as flow and VHT distribution are quite different, which means that each grade of road corresponds to different vehicle driving characteristics. Traffic flow characteristics should be analyzed for different levels of roads, and different transportation policy plans should be formulated.

\subsection{Study on the influence of seasonal turnover on the characteristics of traffic flow}

As the characteristics of people traveling, traffic flow characteristics will be affected by people's willingness to travel. Beijing has a continental monsoon climate with four distinct seasons and obvious differences, which has a certain impact on Beijing automobile users.

It can be found from the seasonal flow charts of various levels of roads in Fig. 7 with seasonal changes that Beijing's overall sample flow shows a regular feature of low winter flow, which is in line with the common sense of traffic flow. The peak time of the morning and evening peaks of the main roads and secondary branches in autumn is earlier than that of 
other peaks in other seasons, but the difference is small. Mainly due to the increasing number of holidays such as the Mid-Autumn Festival National Day in autumn, the weather is gradually cooler, people's work and rest rules have changed, and travel time will be advanced. The peak of autumn evening peaks is higher than the peak of evening peaks of other seasons, of which the expressway has the largest fluctuation, $10.76 \%$. The influence of different seasons on the traffic flow of night peaks of various grades from strong to weak is followed by highways $>$ secondary branch $>$ main roads $>$ Freeway.
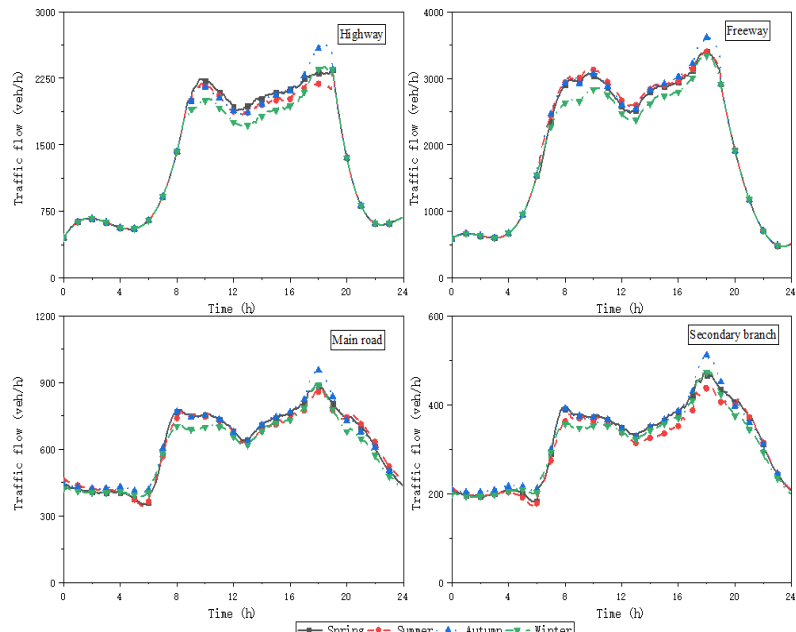

Fig. 7. Four seasons flow chart of various levels of roads.

It can be found from Fig. 8 that the distribution of VHT in the four seasons in Beijing is relatively close. From the average speed, $34.17 \mathrm{~km} / \mathrm{h}$ in spring, $34.77 \mathrm{~km} / \mathrm{h}$ in summer, $34.42 \mathrm{~km} / \mathrm{h}$ in autumn, and $34.47 \mathrm{~km} / \mathrm{h}$ in winter. From the perspective of the distribution range (accumulated speed range corresponding to $10 \%$ and $90 \%$ ), it is $22-50 \mathrm{~km} / \mathrm{h}$ in spring, $24-52 \mathrm{~km} / \mathrm{h}$ in summer, $22-50 \mathrm{~km} / \mathrm{h}$ in autumn, and $22-52 \mathrm{~km} / \mathrm{h}$ in winter. Among them, the peak in autumn is higher, the velocity distribution is more concentrated, and the seasonal change has less effect on the velocity distribution.

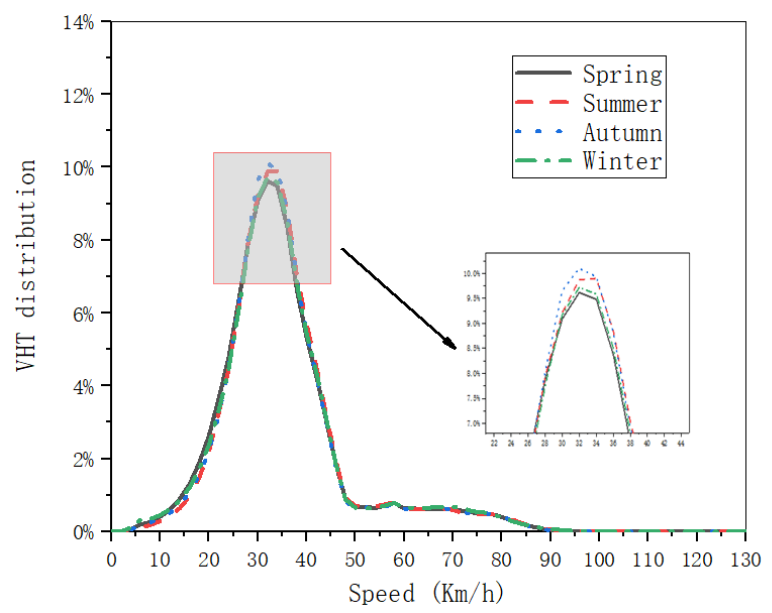

Fig. 8. VHT distribution map of the whole season network. 


\section{Conclusions}

Based on the GPS/GIS low-frequency traffic flow big data, this paper selects the Underwood index model suitable for the low-density state as the traffic flow calculation model, and calibrates the model parameters according to the vehicle driving characteristics of each road class. And made several amendments to the traffic in the early morning. Data mining is carried out on the road data of the whole road network, which mainly analyzes the influence of roads of different grades and seasons on the characteristics of traffic flow. The main conclusions are as follows:

a) The main roads and secondary roads cover more than $80 \%$ of the roads in Beijing's entire road network, and changes in flow have a greater impact on traffic characteristics of the whole road network.

b) The flow trends of all levels of the road network are consistent, the flow timing chart is obviously saddle-shaped, and the flow will drop slightly at noon. The average flow of expressways and expressways is significantly higher than that of main roads and secondary branches.

c) The total flow of roads of all levels shows a trend of high in summer and autumn and low in spring and winter; the autumn climate is cool, the number of holidays increases, and the peak time of the morning and evening peaks is earlier than other seasons; Change, the fluctuation range is between $5 \%-11 \%$.

d)The traffic flow speed distribution of each road network is skewed, and the VHT distribution characteristics of the subdivision speed sections of various levels of roads vary greatly, and each level of road corresponds to different vehicle driving characteristics. Seasonal change has little effect on the speed distribution, and the average speed fluctuation range of each season is within $1 \%$ of the annual average speed. Therefore, traffic flow characteristics should be analyzed for different grades of roads, and different traffic policy plans should be formulated.

This work was supported by the National Key R\&D Program (2018YFB0106404).

\section{References}

1. Kwang-Yul Kim, Chae-Young Lim, Eunice J. Kim. A new approach to the space - time analysis of big data: application to subway traffic data in Seoul [J]. Journal of Big Data, 2018, 5(1) .

2. D. Helbing, A. Hennecke, V. Shvetsov, M. Treiber, Micro- and macro-simulation of freeway traffic, Math. Comput. Modelling 35 (2002) 547.

3. M.J. Lighthill, G.B. Whitham, On kinematic waves. ii. a theory of traffic flow on long crowded roads, in: Proceedings of the Royal Society of

4. Lukas Ambühl,Monica Menendez. Data fusion algorithm for macroscopic fundamental diagram estimation[J]. Transportation Research Part C,2016,71.

5. Greenshields B D, Charming W, Miller H. A study of traffic capacity[C]. Highway research board proceedings. National Research Council (USA), Highway Research Board,1935.

6. Greenberg H. An analysis of traffic flow[J]. Operations research, 1959, 7(1): 79-85.

7. Underwood R T. Speed, volume, and density relationships[J]. Yale University Report, Yale Bureau of Highway Traffic, New Haven, CT, pp. 1961, 141188.

8. Transportation Research Board, National Rrsearch Council. Highway Capacity Manual [R].Washington, D.C., 2010. 